\title{
Artigo Original / Original Paper \\ Epífitas vasculares da mata de restinga da Praia do Sul, Ilha Grande, RJ, Brasil
}

Vascular epiphytes of the restinga forest of Praia do Sul, Ilha Grande, RJ, Brazil

\author{
Ana Carolina Rodrigues da Cruz $^{1,2}$ \& André Felippe Nunes-Freitas ${ }^{1}$
}

\begin{abstract}
Resumo
Plantas epífitas apresentam elevada riqueza e importância ecológica em ecossistemas de Mata Atlântica. O presente estudo visa contribuir para o conhecimento da flora de epífitas vasculares da mata de restinga na Praia do Sul, Ilha Grande, RJ. Demarcamos 52 subparcelas de $100 \mathrm{~m}^{2}$ na Reserva Biológica Estadual da Praia do Sul (RBEPS), onde todas as árvores foram vistoriadas e tiveram suas epífitas registradas. Foram identificadas 31 espécies de epífitas vasculares, 21 gêneros e nove famílias, sendo nove novos registros para a ilha. As angiospermas foram responsáveis por $80,6 \%$ das espécies. Araceae e Philodendron foram família e gênero com maior riqueza $(22,6 \%$ e 16,1\%, respectivamente). A principal categoria ecológica em relação à fidelidade ao substrato é holoepífita (74,2\%), a forma de polinização é a entomofilia (64\%) e de dispersão, a zoocoria (58,1\%). A maior parte das espécies é endêmica da Mata Atlântica (63\%) e possui status de conservação desconhecido (74\%). Este é o primeiro estudo sobre epífitas vasculares na Praia do Sul e destaca a importância da preservação da área de estudo para a conservação das epífitas vasculares da Ilha Grande e do Rio de Janeiro. Palavras-chave: biodiversidade, conservação, ecologia, Mata Atlântica, polinização.
\end{abstract}

\begin{abstract}
Epiphytic plants show high richness and ecological importance in Atlantic Forest ecosystems. The present study aims to contribute to the knowledge of the vascular epiphyte flora of the restinga forest in Praia do Sul, Ilha Grande, RJ. We demarcated 52 subplots of $100 \mathrm{~m}^{2}$ in the Praia do Sul State Biological Reserve (RBEPS), inspected all the trees and recorded all the epiphytes found. We identified 31 species of vascular epiphytes, 21 genera and nine family, with nine new records for the island. The angiosperms were responsible for $80.6 \%$ of the species. Araceae and Philodendron were the family and genus with greatest wealth $(22.6 \%$ and $16.1 \%$, respectively). The main ecological category in relation to substrate fidelity is holoepiphyte, the form of pollination is entomophilia (64\%) and dispersion is zoocoria (58.1\%). Most species are endemic to the Atlantic Forest (63\%) and have an unknown conservation status (74\%). This is the first study on vascular epiphytes in Praia do Sul and highlights the importance of preserving the study area for the conservation of the vascular epiphytes of Ilha Grande and Rio de Janeiro.
\end{abstract}

Key words: biodiversity, conservation, ecology, Atlantic Forest, pollination.

\section{Introdução}

Epífitas são plantas que utilizam outras espécies (em geral árvores e arbustos) como suporte mecânico por todo ou em alguma parte do seu ciclo de vida (Madison 1977). O epifitismo está presente em mais de 27 mil espécies de plantas, incluindo hemiepífitas primárias, são 913 gêneros de 73 famílias, representando cerca de $9 \%$ de todas as plantas vasculares do planeta (Zotz 2013). Em escala mundial, as famílias mais ricas em espécies epifíticas são Orchidaceae, Araceae e Bromeliaceae (Madison 1977). Epífitas ocorrem principalmente nas Florestas Neotropicais, onde são muito ricas e abundantes (Gentry \& Dodson 1987; Nieder et

\footnotetext{
${ }^{1}$ Universidade Federal Rural do Rio de Janeiro, Inst. Florestas, Depto. Ciências Ambientais, Lab. Ecologia Florestal e Biologia Vegetal, BR-465, km 7, Campus Universitário, Seropédica, RJ, Brasil.

22Autor para correspondência: anacarolina091087@yahoo.com.br
} 
al. 1999). No domínio Amazônico destacam-se Araceae e Orchidaceae como as famílias de maior riqueza de espécies (Benavides et al. 2005; Irume et al. 2013; Pos \& Sleegers 2010; Obermuller et al. 2014; Quaresma \& Jardim 2014), enquanto no domínio Atlântico sobressaem Orchidaceae e Bromeliaceae na maioria dos levantamentos (e.g., Kersten 2010).

A Mata Atlântica corresponde a uma das regiões do mundo onde o hábito epifítico é altamente pronunciado (Freitas et al. 2016), juntamente com as Américas Central e do Sul e o cinturão subandino (Nieder et al. 1999). É possível estimar mais de 3.000 espécies ocorrendo em seus ecossistemas (Kersten 2010), sendo as florestas do Rio de Janeiro e do Espírito Santo as que apresentam maiores riquezas e taxas de endemismos (Menini Neto et al. 2016). As epífitas apresentam grande importância taxonômica e ecológica nos ambientes, uma vez que sua riqueza corresponde a $15 \%$ da flora vascular conhecida da Mata Atlântica (Freitas et al. 2016) e a mais de $50 \%$ das espécies em algumas áreas (Kersten \& Silva 2006). A comunidade epifítica gera diversificação de nichos e habitats no dossel, servindo como abrigo, refúgio reprodutivo e fonte de alimento para vários animais (Cruz-Angón \& Greenberg 2005; Sabagh et al. 2011; Dias et al. 2014), bem como participando de importantes processos ecossistêmicos, com elevada produção de serapilheira (Nadkarni \& Matelson 1992) e fixação de nitrogênio (Puente \& Bashan 1994).

As restingas, comunidades que recebem influência direta da água do mar, são compreendidas como o conjunto formado pela deposição de sedimentos arenosos marinhos quaternários e toda a biota integrada (Araujo \& Lacerda 1987; Veloso et al. 1991). Essas formações constituem um dos mais importantes ecossistemas da Mata Atlântica e podem apresentar diferentes tipos vegetacionais, formações herbáceas, arbustivas e florestais (Araujo 2000; Thomazi et al. 2013), e são associadas a uma elevada biodiversidade (Araujo 2000; Assis et al. 2004; Rocha et al. 2003; Martins et al. 2008). As restingas se estabelecem sobre solos arenosos, pobres em nutrientes e altamente lixiviados e tornam-se, por esta razão, ambientes extremamente frágeis e passíveis de perturbações (Guedes et al. 2006). Como os demais ecossistemas costeiros, sofrem com grande pressão antrópica devido à ocupação do país, que se deu da zona costeira para o interior, sendo, até hoje, a urbanização a sua principal ameaça (Cunha 2005; Rocha et al. 2004). As restingas vêm sendo destruídas sem que se tenha adquirido o conhecimento sobre elas, carecendo de dados sobre riqueza florística e estrutura das comunidades (Thomazi et al. 2003).

O estado do Rio de Janeiro conta com 21 remanescentes de restingas, totalizando mais de 60.000 ha inseridos em áreas que passam por acelerado processo de urbanização (Rocha et al. 2007). As restingas fluminenses abrigam 1.513 espécies de plantas vasculares, distribuídas em 170 famílias (Brazil Flora Group 2018; Flora do Brasil 2020), das quais se destacam Fabaceae, Myrtaceae, Bromeliaceae, Rubiaceae e Orchidaceae como as mais ricas em espécies (Araujo 2000). O conhecimento sobre a flora epifítica nas restingas é reduzido em comparação a outros ecossistemas de Mata Atlântica e conta com um menor número de trabalhos publicados, sendo a maioria concentrados na região sul do Brasil (Waechter 1986, 1992, 1998; Kersten \& Silva 2001; Musskopf 2006; Kersten \& Silva 2006; Martins et al. 2008; Fontoura et al. 2009; Kersten et al. 2009; Ribeiro 2009; Mania \& Monteiro 2010; Staudt et al. 2012; Becker et al. 2013; Graeff et al. 2015; Couto et al. 2017). A Reserva Biológica Estadual da Praia do Sul (RBEPS), que compreende florestas e restingas na Ilha Grande é uma importante área de preservação do Rio de Janeiro (Rocha et al. 2007) e possui apenas um inventário preliminar da flora publicado por Araujo \& Oliveira (1988) e um levantamento da família Bromeliaceae (Nunes-Freitas et al. 2006). Assim, há muitas lacunas de conhecimento para as famílias e comunidades vegetais na RBEPS. Diante da relevância das epífitas vasculares nos ecossistemas e da escassez de pesquisas na área, o presente estudo levanta e avalia aspectos florísticos e ecológicos das epífitas da mata de restinga da RBEPS, bem como analisa dados referentes a endemismos e conservação das espécies.

\section{Material e Métodos}

Área de estudo

O estudo foi realizado na Reserva Biológica da Praia do Sul (RBEPS), localizada na vertente oceânica da Ilha Grande, maior ilha do litoral do estado do Rio de Janeiro e que possui um dos mais importantes remanescentes de Mata Atlântica do sudeste brasileiro (Alho et al. 2002). A ilha apresenta Floresta Ombrófila Densa, matas secundárias, formações herbáceas, herbáceas de afloramentos rochosos, restingas, manguezais e praias, configurando uma elevada diversidade de habitats (Alho et al. 2002). O relevo é acidentado, com muitas montanhas e planícies costeiras (INEA 
2011). O clima é Tropical Úmido Af de Köppen, quente e úmido, sem estação seca e com temperatura média anual de $21^{\circ} \mathrm{C}$, com umidade do ar em torno de 80 a 95\% (INEA 2011). A precipitação na ilha é desigual devido ao relevo bastante acidentado e pode atingir até $4.500 \mathrm{~mm}$ de chuva em algumas regiões (Oliveira \& Coelho Netto 2001), com média anual de $2.242 \mathrm{~mm}$ nas baixadas próximas ao nível do mar, sendo janeiro o mês mais chuvoso e julho o mais seco (INEA 2011).

A RBEPS é uma Unidade de Conservação de Proteção Integral criada pelo Decreto Estadual $n^{\circ}$ 4.972, de 2 de dezembro de 1981 e atualmente conta com 3.502 hectares (INEA 2017). A composição florística da RBEPS varia devido a sua grande diversidade de condições geomorfológicas, apresentando praia, restinga, depósitos lagunares e aluviais, sedimentos de pântanos e manguezais (Araujo \& Oliveira 1988). Araujo \& Oliveira (1988) reconhecem quatro comunidades vegetais para a RBEPS: manguezal, psamófila reptante da anteduna, mata alagadiça e de cordão arenoso. A mata de cordão arenoso, conhecida como mata de restinga, cobre o cordão externo da restinga e constitui uma floresta baixa com cerca de $10 \mathrm{~m}$ de altura (Araujo \& Oliveira 1988). A copa das árvores é contínua, mas permite passagem de luz suficiente para o estabelecimento de um estrato inferior rico em espécies de várias famílias botânicas, especialmente Araceae e Bromeliaceae (Araujo \& Oliveira 1988; Nunes-Freitas et al. 2006).

\section{Metodologia}

Foram utilizadas as parcelas permanentes do RAPELD-Ilha Grande instaladas na mata de restinga na RBEPS. O método RAPELD é adotado pelo Programa de Pesquisas em Biodiversidade (PPBio) e a sigla resulta da união das duas escalas que o sistema abrange: inventários rápidos (RAP) e projetos ecológicos de longa duração (PELD) (para maiores detalhes veja Magnusson et al. 2005). Foram demarcadas 52 subparcelas de $100 \mathrm{~m}^{2}(10$ $\mathrm{m} \times 10 \mathrm{~m}$ ) de ambos os lados do corredor central das parcelas, intercalando-se segmentos de $10 \mathrm{~m}$. Nas subparcelas, todas as árvores foram vistoriadas e tiveram as espécies de epífitas registradas por meio de coletas ou fotografias (aquelas cuja coleta não foi possível). O material botânico foi coletado com tesoura de poda e com o auxílio de um podão telescópico de $5 \mathrm{~m}$. As coletas foram realizadas nos meses de julho e outubro de 2016 e todo o material coletado recebeu procedimentos de herborização usuais e depositado no Herbário
RBR da Universidade do Federal Rural do Rio de Janeiro (UFRRJ). A identificação das espécies foi baseada em literatura específica, no estudo de material herborizado e com auxílio de especialistas. Os nomes científicos foram verificados em International Plant Names Index (IPNI) e em Brazil Flora Group (BFG 2018). Espécies não levantadas por Araujo \& Oliveira (1988) e que não constam registradas em bancos de dados online (speciesLink, JABOT - Banco de Dados da Flora Brasileira e Catálogo da Flora do Rio de Janeiro) foram consideradas novos registros para a área.

As espécies levantadas foram classificadas em categorias ecológicas, quanto à dependência do substrato, em holoepífitas e hemiepífitas, segundo Benzing (1990). As holoepífitas são espécies que apresentam epifitismo durante todo o ciclo de vida e podem ser verdadeiras, facultativas ou acidentais (Benzing 1990). As hemiepífitas são plantas que apresentam o epifitismo durante parte de seu ciclo de vida e podem ser primárias ou secundárias (Benzing 1990). A classificação das espécies registradas foi baseada em consultas a bibliografia específica e em observações feitas na área de estudo.

As síndromes de polinização das espécies foram baseadas em Real (1983) e Proctor et al. (1996) e de dispersão em Gentry \& Dodson (1987). As informações foram levantadas a partir de características morfológicas das espécies e de consultas a bibliografias específicas, não foram realizadas pesquisas aprofundadas sobre a biologia reprodutiva das espécies. As formas de polinização das angiospermas foram classificadas de acordo com as síndromes florais e categorizadas em: anemofilia, quando ocorre através do vento; entomofilia, por meio de insetos; ornitofilia, de aves; e quiropterofilia, quando se dá por morcegos. Quanto à dispersão de diásporos, as espécies foram classificadas em zoocóricas e anemocóricas. A anemocoria pode ser dividida em esporocoria, quando possuem esporos/sementes diminutos, e pogonocoria, quando apresentam sementes plumosas ou aladas (Gentry \& Dodson 1987).

Para verificar se as espécies apresentam distribuição ampla ou restrita à Mata Atlântica foi utilizado o portal da Flora do Brasil 2020 e Brazil Flora Group (BFG 2018). O estado de conservação das espécies foi verificado na Lista Oficial de Espécies da Flora Ameaçadas de Extinção (MMA 2014) e as informações adicionais foram levantadas de acordo com o Livro Vermelho da Flora do Brasil (Martinelli \& Moraes 2013) e o portal do Centro Nacional de Conservação da Flora (CNCFlora 2017). 


\section{Resultados}

Foram registradas 31 espécies de epífitas vasculares, pertencentes a 21 gêneros e nove famílias botânicas na mata de restinga da RBEPS (Tab. 1). Quatro espécies encontravam-se estéreis e não puderam ser identificadas em nível específico.

Nove espécies com identificações confirmadas consistem em novos registros para a Ilha Grande: Anthurium intermedium Kunth, Codonanthe devosiana Lem., Hylocereus setaceus (Salm-Dyck)
R.Bauer, Microgramma crispata (Fée) R.M.Tryon \& A.F.Tryon, Microgramma vacciniifolia (Langsd. \& Fisch.) Copel., Monstera adansonii var. klotzschiana (Schott) Madison, Peperomia urocarpa Fisch. \& C.A.Mey., Philodendron propinquun Schott e Serpocaulon triseriale (Sw.) A.R.Sm. A espécie $M$. vacciniifolia é bastante frequente e abundante na ilha, porém não foram encontrados registros anteriores em herbários ou publicações.

Tabela 1 - Famílias e espécies de epífitas vasculares registradas na mata de restinga da Reserva Biológica Estadual da Praia do Sul, RJ, seguidas de categoria ecológica, de acordo com a fidelidade ao substrato (Benzing 1990), síndrome de polinização, síndrome de dispersão (Gentry \& Dodson 1987), estado de conservação das espécies (Martinelli \& Moraes 2013; MMA 2014; CNCFlora 2017) e acrônimo com número de registro do herbário no qual encontramse depositadas. $\mathrm{CE}=$ categorias ecológicas; $\mathrm{SP}=$ síndromes de polinização; $\mathrm{SD}=$ síndromes de dispersão; HLV = holoepífita verdadeira; HLF = holoepífita facultativa; HLA = holoepífita acidental; HEP = hemiepífita primária; HES = hemiepífita secundária; $\mathrm{ANE}=$ anemofilia; $\mathrm{ENT}=$ entomofilia; $\mathrm{ORN}=$ ornitofilia; $\mathrm{ESP}=$ esporocoria; $\mathrm{POG}$ = pogonocoria; $\mathrm{ZOO}=$ zoocoria; $\mathrm{NE}=$ não avaliada $\mathrm{LC}=$ pouco preocupante; $\mathrm{RBR}=$ Herbário do Departamento de Botânica da Universidade Federal Rural do Rio de Janeiro; $\mathrm{NC}$ = material não coletado.

Table 1 - Families and species of vascular epiphytes recorded in the restinga forest of the State Biological Reserve of Praia do Sul, RJ, followed by ecological category, according to substrate fidelity (Benzing 1990), pollination syndrome, dispersion syndrome Gentry \& Dodson 1987), species conservation status (Martinelli \& Moraes 2013, MMA 2014 and CNCFlora 2017) and acronym with registration number of the herbarium in which they are deposited. $\mathrm{EC}=$ ecological categories; $\mathrm{SP}=$ pollination syndromes; $\mathrm{SD}=$ dispersion syndromes; HLV = true holoepífita; HLF = optional holoepífita; HLA = accidental holoepífita; HEP = primary hemiepiphyte; HES = secondary hemiepiphyte; $\mathrm{ANE}=$ anemophilia; $\mathrm{ENT}=$ entomophilia; $\mathrm{ORN}=$ ornithophilia; $\mathrm{ESP}=$ sporocoria; $\mathrm{POG}=$ pogonocoria; $\mathrm{ZOO}$ = zoocoria; $\mathrm{NE}=$ not evaluated; $\mathrm{LC}=$ slightly worrisome; $\mathrm{RBR}=$ Herbarium of the Department of Botany of the Federal Rural University of Rio de Janeiro; $\mathrm{NC}=$ uncollected material.

\begin{tabular}{llccccc}
\hline Família & Espécies & CE & SP & SD & $\begin{array}{c}\text { cstado de } \\
\text { Conservação }\end{array}$ & $\begin{array}{c}\text { Acrônimo e } \\
\text { número de } \\
\text { registro em } \\
\text { herbário }\end{array}$ \\
\hline Araceae & Anthurium intermedium Kunth & & & & NE & RBR 42711 \\
Araceae & $\begin{array}{l}\text { Monstera adansonii var. } \text { klotzschiana } \\
\text { (Schott) Madison }\end{array}$ & HES & ENT & ZOO & NE & RBR 42693 \\
Araceae & Philodendron cordatum Kunth ex Schott & HEP & ENT & ZOO & NE & RBR 42726 \\
Araceae & Philodendron crassinervium Lindl. & HEP & ENT & ZOO & NE & RBR 42703 \\
Araceae & Philodendron curvilobum Schott & HES & ENT & ZOO & NE & RBR 42715 \\
Araceae & Philodendron propinquum Schott & HMS & ENT & ZOO & NE & NC \\
Araceae & Philodendron martianum Engl. & HLF & ENT & ZOO & NE & NC \\
Bromeliaceae & Aechmea nudicaulis (L.) Griseb. & HLF & ORN & ZOO & LC & RBR 42714 \\
Bromeliaceae & Billbergia amoena (Lodd.) Lindl. & HLF & ORN & ZOO & NE & RBR 42713 \\
Bromeliaceae & Neoregelia johannis (Carrière) L.B.Sm. & HLF & ORN & ZOO & NE & RBR 42701 \\
Bromeliaceae & Tillandsia dura Baker & HLV & ORN & POG & NE & RBR 42716 \\
Bromeliaceae & Vriesea rodigasiana E.Morren & HLF & ORN & POG & LC & RBR 42704 \\
\hline
\end{tabular}




\begin{tabular}{|c|c|c|c|c|c|c|}
\hline Família & Espécies & $\mathbf{C E}$ & SP & SD & $\begin{array}{c}\text { Estado de } \\
\text { Conservação }\end{array}$ & $\begin{array}{l}\text { Acrônimo e } \\
\text { número de } \\
\text { registro em } \\
\text { herbário }\end{array}$ \\
\hline Cactaceae & Hylocereus setaceus (Salm-Dyck) R.Bauer & HLV & QUI & $\mathrm{ZOO}$ & LC & RBR 42722 \\
\hline Cactaceae & Rhipsalis pachyptera Pfeiff. & HLV & ENT & $\mathrm{ZOO}$ & NE & RBR 42707 \\
\hline Cactaceae & Rhipsalis sp. & HLV & ENT & $\mathrm{ZOO}$ & - & RBR 42699 \\
\hline Gesneriaceae & Codonanthe devosiana Lem. & HLV & ENT & $\mathrm{ZOO}$ & $\mathrm{LC}$ & RBR 42718 \\
\hline Gesneriaceae & Codonanthe gracilis (Mart.) Hanst. & HLV & ENT & $\mathrm{ZOO}$ & $\mathrm{LC}$ & RBR 42698 \\
\hline Iridaceae & Neomarica candida (Hassl.) Sprague & HLA & ENT & POG & NE & RBR 42724 \\
\hline Lycopodiaceae & Phlegmariurus cf. mandiocanus (Raddi) B.Øllg. & HLV & - & ESP & $\mathrm{NE}$ & $\mathrm{NC}$ \\
\hline Orchidaceae & Acianthera sp. & HLV & ENT & ESP & - & RBR 42697 \\
\hline Orchidaceae & Cattleya forbesii Lindl. & HLV & ENT & ESP & NE & RBR 42719 \\
\hline Orchidaceae & Octomeria cf. grandiflora Lindl. & HLV & ENT & ESP & $\mathrm{NE}$ & RBR 42720 \\
\hline Orchidaceae & Vanilla chamissonis Klotzsch & HES & ENT & ESP & $\mathrm{LC}$ & RBR 42694 \\
\hline Piperaceae & Peperomia urocarpa Fisch. \& C.A.Mey. & HLV & ANE & $\mathrm{ZOO}$ & NE & RBR 42695 \\
\hline Piperaceae & Peperomia sp.1 & HLV & ANE & $\mathrm{ZOO}$ & - & $\mathrm{NC}$ \\
\hline Piperaceae & Peperomia sp.2 & HLV & ANE & $\mathrm{ZOO}$ & - & $\mathrm{NC}$ \\
\hline Polypodiaceae & $\begin{array}{l}\text { Microgramma crispata } \\
\text { (Fée) R.M.Tryon \& A.F.Tryon }\end{array}$ & HLV & - & ESP & $\mathrm{LC}$ & RBR 42708 \\
\hline Polypodiaceae & $\begin{array}{l}\text { Microgramma geminata } \\
\text { (Schrad.) R.M.Tryon \& A.F.Tryon }\end{array}$ & HLV & - & ESP & $\mathrm{NE}$ & RBR 42706 \\
\hline Polypodiaceae & $\begin{array}{l}\text { Microgramma vacciniifolia } \\
\text { (Langsd. \& Fisch.) Copel. }\end{array}$ & HLV & - & ESP & NE & RBR 42710 \\
\hline Polypodiaceae & Pleopeltis pleopeltifolia (Raddi) Alston & HLV & - & ESP & NE & RBR 42709 \\
\hline Polypodiaceae & Serpocaulon triseriale (Sw.) A.R.Sm. & HLF & - & ESP & $\mathrm{NE}$ & RBR 42727 \\
\hline
\end{tabular}

As angiospermas foram responsáveis por 25 espécies $(80,6 \%), 17$ gêneros e sete famílias, enquanto que as pteridófitas contribuíram com seis espécies $(19,4 \%)$, quatro gêneros e duas famílias. Araceae é a família botânica mais representativa, com sete espécies (22,6\%), seguida por Bromeliaceae e Polypodiaceae, com cinco espécies cada (16,1\%) (Fig. 1). O gênero com maior número de espécies é Philodendron Schott (Araceae), com cinco espécies (16,1\%), seguido por Microgramma C.Presl (Polypodiaceae) e Peperomia Ruiz \& Pav. (Piperaceae), com três espécies cada $(9,7 \%)$.

A classificação das espécies em categorias ecológicas demonstrou um predomínio de

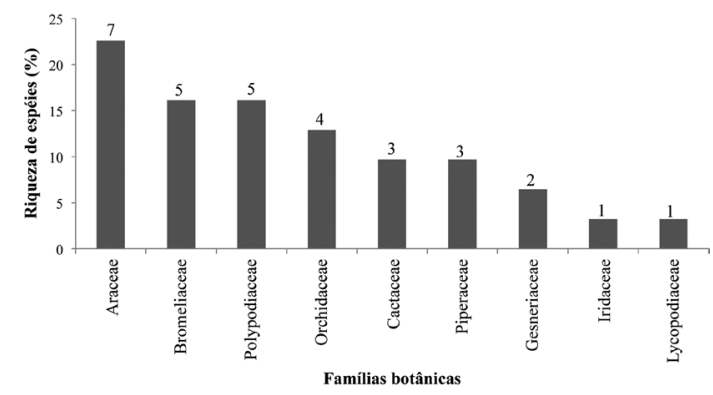

Figura 1 - Riqueza de espécies de epífitas vasculares por família botânica da mata de restinga da Reserva Biológica Estadual da Praia do Sul, Ilha Grande, RJ. Figure 1 - Richness of vascular epiphyte species by botanical family of the restinga forest of the Praia do Sul State Biological Reserve, Ilha Grande, RJ. 
holoepífitas ( 23 espécies, 74,2\%). As hemiepífitas somaram 19,4\% do total (seis espécies) e duas espécies, Anthurium intermedium Kunth e Neomarica candida (Hassl.) Sprague, foram consideradas holoepífitas acidentais $(6,5 \%)$ (Fig. 2).

A maioria das espécies é polinizada por animais, sendo a entomofilia a principal síndrome de polinização (16 espécies; 64\%), seguida pela ornitofilia (5 espécies; 20\%) (Fig. 3). A principal forma de dispersão é a zoocoria (18 espécies; $58,1 \%$ ), sendo que as espécies que apresentaram dispersão pelo vento (esporocoria e pogonocoria) somaram 41,9\% (13 espécies) (Fig. 3).

Nove espécies apresentam ampla distribuição geográfica e ocorrem em dois ou mais domínios fitogeográficos (33,3\%). A maior parte das espécies é restrita à Mata Atlântica (17 espécies; 63\%), sendo que uma delas, Neoregelia johannis (Carrière) L.B.Sm., é endêmica da Região Sudeste nos estados do Rio de Janeiro e São Paulo.

Nenhuma das espécies inventariadas consta no Livro Vermelho da Flora do Brasil (Martinelli \& Moraes 2013) ou na Lista Oficial de Espécies da Flora Ameaçadas de Extinção (MMA 2014). De acordo com Centro Nacional de Conservação da Flora (CNCFlora 2017), 20 espécies não foram avaliadas $(\mathrm{NE}=74 \%)$ e sete foram classificadas em situação pouco preocupante $(\mathrm{LC}=26 \%)$ (Tab. 1).

Foram levantadas 140 espécies de epífitas vasculares, incluindo hemiepífitas, para as restingas do estado do Rio de Janeiro, 127 angiospermas e 13 samambaias e licófitas (BFG 2018; Flora do Brasil 2020) e a riqueza da RBEPS pode ser comparada a outros trabalhos realizados em restingas (Tab. 2).

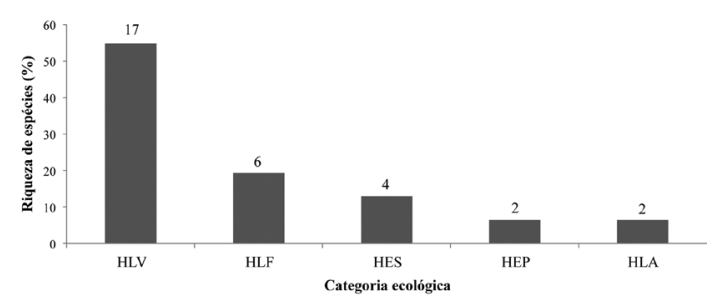

Figura 2 - Riqueza de espécies de epífitas vasculares da mata de restinga da Reserva Biológica Estadual da Praia do Sul, RJ, por categoria ecológica, quanto à fidelidade ao uso do substrato (Benzing 1990). HLV = holoepífitas verdadeiras; HLF = holoepífitas facultativas; HLA = holoepífitas acidentais; HEP = hemiepífitas primárias; HES $=$ hemiepífitas secundárias.

Figure 2 - Richness of vascular epiphyte species from the restinga forest of the State Biological Reserve of Praia do Sul, $\mathrm{RJ}$, by ecological category, regarding the fidelity to the use of the substrate (Benzing 1990). HLV = true holoepífitas; HLF = optional holoepífitas; HLA = accidental holoepífitas; HEP $=$ primary hemiepiphytes; HES $=$ secondary hemiepiphytes.

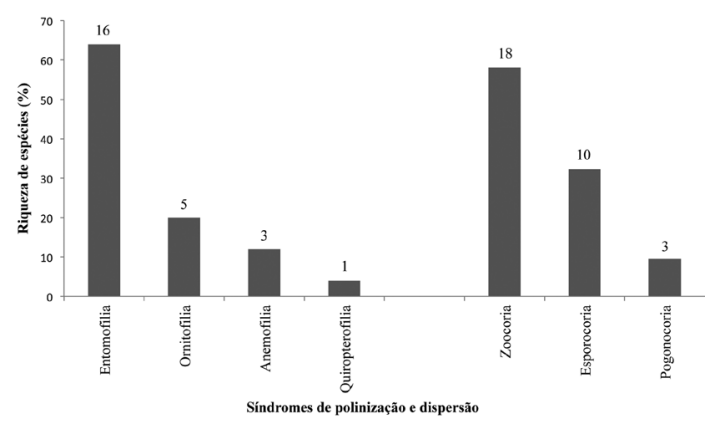

Figura 3 - Síndromes de polinização e dispersão das espécies de epífitas vasculares registradas na mata de restinga da Reserva Biológica Estadual da Praia do Sul, RJ.

Figure 3 - Pollination and dispersion syndromes of the vascular epiphyte species recorded in the restinga forest of the State Biological Reserve of Praia do Sul, RJ.

Tabela 2 - Comparação da riqueza de espécies de epífitas vasculares em fitofisionomias de restinga nas regiões sul e sudeste do Brasil.

Table 2 - Comparison of species richness of vascular epiphytes in restinga phytophysiognomies in southern and southeastern Brazil.

\begin{tabular}{lllc}
\hline Referência & Localidade & Fitofisionomia & Riqueza \\
\hline Flora do Brasil 2020 & Estado do Rio de Janeiro & Restingas & 140 \\
Breier (2005) & $\begin{array}{l}\text { Parque Estadual da Ilha do Cardoso, } \\
\text { município de Cananéia (SP) }\end{array}$ & Restinga & 178 \\
Kersten \& Silva (2006) & Ilha do Mel (PR) & Floresta inundável & 103 \\
Martins et al. (2008) & $\begin{array}{l}\text { Itaguaré, São Lourenço e Guaratuba, } \\
\text { município de Bertioga (SP) }\end{array}$ & Restinga & 96 \\
\hline
\end{tabular}




\begin{tabular}{lllc}
\hline Referência & Localidade & Fitofisionomia & Riqueza \\
\hline Kersten \& Silva (2001) & Ilha do Mel, município de Paranaguá (PR) & Floresta não hidrófila de restinga & 77 \\
Mania \& Monteiro (2010) & $\begin{array}{l}\text { Parque Estadual da Serra do Mar, município } \\
\text { de Ubatuba (SP) }\end{array}$ & Floresta alta de restinga & 64 \\
Musskopf (2006) & Parque Estadual de Itapuã (RS) & Floresta de encosta e de restinga \\
Staudt et al. (2012) & $\begin{array}{l}\text { Parque Natural Municipal de Tupancy, } \\
\text { Município de Arroio do Sal (RS) }\end{array}$ & Floresta Psamófila \\
Couto et al. (2017) & Área de Proteção Ambiental Grumari (RJ) & Restinga \\
Fontoura et al. (2009) & $\begin{array}{l}\text { Reserva Ecológica Estadual de Jacarepiá, } \\
\text { município de Saquarema (RJ) }\end{array}$ & Floresta seca \\
Waechter (1998) & Emboaba, município de Osório (RS) & Floresta de restinga \\
$\begin{array}{l}\text { Cruz \& Nunes- Freitas } \\
\text { (presente estudo) }\end{array}$ & $\begin{array}{l}\text { Ilha Grande, município de Angra dos Reis } \\
\text { (RJ) }\end{array}$ & Mata de restinga \\
Becker et al. (2013) & $\begin{array}{l}\text { Parque Natural Municipal de Tupancy, } \\
\text { Município de Arroio do Sal (RS) }\end{array}$ & Floresta Psamófila \\
Graeff et al. (2015) & $\begin{array}{l}\text { Fragmento no município de Palmares do Sul } \\
\text { (RS) }\end{array}$ & Floresta de restinga \\
\hline
\end{tabular}

\section{Discussão}

A maior parte das epífitas na RBEPS é angiosperma, seguindo o padrão mundial para o grupo (Madison 1977; Benzing 1990; Kersten 2010; Freitas et al. 2016). No entanto, a família mais representativa, Araceae, difere da maioria dos estudos realizados em ecossistemas de Mata Atlântica, bem como em restingas, onde geralmente se destaca a família Orchidaceae como a mais rica (Fontoura et al. 1997; Waechter 1998; Kersten \& Silva 2001; Gonçalves \& Waechter 2002; Giongo \& Waechter 2004; Hefler \& Faustioni 2004; Breier 2005; Kersten \& Silva 2006; Martins et al. 2008; Fontoura et al. 2009; Kersten \& Kuniyoshi 2009; Mania \& Monteiro 2010; Bianchi et al. 2012; Staudt et al. 2012; Perleberg et al. 2013; Graeff et al. 2015; Freitas et al. 2016; Couto et al. 2017). Araceae foi seguida por Bromeliaceae e Polypodiaceae em número de espécies, duas das famílias com maior riqueza de epífitas na Mata Atlântica (Kersten 2010; Freitas et al. 2016). As bromélias, em especial, se destacam em número de espécies em levantamentos de epífitas (Dettke et al. 2008; Freitas \& Assis 2013; Freitas et al. 2016), inclusive em restingas (Staudt et al. 2012; Becker et al. 2013; Couto et al. 2017). O hábito epifítico é bastante comum na família e vários gêneros possuem adaptações para esse modo de vida, como capacidade de armazenar água e de absorver nutrientes pelos tricomas de suas folhas
(Benzing 1990, 2000). Na mata de restinga da RBEPS há uma elevada riqueza de bromélias (Araujo \& Oliveira 1988; Nunes-Freitas et al. 2006), o que, de acordo Nunes-Freitas et al. (2006), está relacionado à presença da floresta de encosta adjacente às formações de restinga, permitindo que espécies da Floresta Ombrófila Densa também ocorram na restinga.

A família Araceae é uma das mais importantes nos Neotrópicos (Coelho et al. 2009) e está muito bem representada na RBEPS, tanto em espécies terrícolas quanto hemiepífitas (Araujo \& Oliveira 1988). Araceae apresenta elevada riqueza em florestas de baixas altitudes (Krömer et al. 2007), como as florestas de baixada e submontana, que formam um contínuo com as florestas de restinga da RBEPS, permitindo que um grande número de espécies dessas formações seja encontrado nas matas de restinga da área. Resultados semelhantes para a comunidade de epífitas foram registrados nas florestas da Amazônia brasileira e colombiana (Benavides et al. 2005; Irume et al. 2013; Pos \& Sleegers 2010; Obermuller et al. 2014; Quaresma \& Jardim 2014).

A baixa riqueza de orquídeas levantada pode ser um reflexo da ação antrópica ocorrida em alguns trechos da mata de restinga no passado, quando esta foi utilizada para agricultura (Araujo \& Oliveira 1988). A distribuição do número de espécies entre as famílias de epífitas é modificada 
em vegetações secundárias (Barthlott et al. 2001; Krömer et al. 2007; Bataghin et al. 2008; Adhikari et al. 2012), com a perda local de algumas famílias mais sensíveis, tais como Orchidaceae (Dislich \& Mantovani 1998; Adhikari et al. 2012). Além disso, o tempo de regeneração da mata pode ainda não ter sido suficiente para a recolonização por orquídeas epífitas, que levam mais tempo para colonizar esses ambientes devido às maiores exigências ecofisiológicas e por limitações na dispersão (Dislich \& Mantovani 1998; Barthlott et al. 2001; Krömer et al. 2007; Adhikari et al. 2012). A interferência humana causa alterações no dossel da floresta e leva a mudanças na luminosidade e umidade, impactando diretamente a sobrevivência e colonização de orquídeas (Adhikari et al. 2012). Já para Araceae, cujas espécies possuem alta variabilidade morfológica e fisiológica (Coelho et al. 2009) e apresentam grande capacidade de colonização e propagação, estes fatores parecem não ter efeito (Benavides et al. 2006; Krömer et al. 2007), o que possibilita a família desenvolver elevada diversidade nesses ambientes (Irume et al. 2013).

O gênero Philodendron, o mais expressivo na área de estudo, é um gênero exclusivamente Neotropical (Mayo 1988; Sakuragui 2001) e é bem representado na flora epifítica da Mata Atlântica (Kersten 2010). O gênero é nativo, não endêmico do Brasil e com a segunda maior riqueza na família Araceae, são mais de 350 espécies reconhecidas com mais de $80 \%$ delas epífitas ou hemiepífitas (Benzing 1990). Philodendron é bem diversificado nas florestas tropicais (Mayo 1988) e a maior diversidade ocorre nos estados do Amazonas, Espírito Santo e Rio de Janeiro (Sakuragui 2001). Na Mata Atlântica ocorrem 168 espécies de Philodendron, com oito epífitas e 54 hemiepífitas (BFG 2018; Flora do Brasil 2020). No Rio de Janeiro são registradas 28 hemiepífitas e duas espécies epífitas, $P$ crassinervium e $P$. martianum (Coelho et al. 2015), ambas registradas na restinga da Praia do Sul. Segundo Araujo (2000), Philodendron é um dos gêneros de maior riqueza nas restingas do estado.

A classificação das epífitas em categorias ecológicas fornece uma maneira prática para se compreender a dependência das epífitas em relação ao substrato (Burns 2010). Na RBEPS há um predomínio de holoepífitas, como o padrão observado em comunidades na Mata Atlântica e em outras florestas no mundo (Fontoura et al. 1997; Nieder et al. 2000; Kersten \& Silva 2001;
Kersten \& Silva 2002; Hefler \& Faustioni 2004; Cervi \& Borgo 2007; Dettke et al. 2008; Kersten \& Kuniyoshi 2009; Kersten et al. 2009; Burns 2010; Mania \& Monteiro 2010; Bataghin et al. 2010, 2017; Blum et al. 2011; Bianchi et al. 2012; Staudt et al. 2012). O grau de especialização evolutiva para o epifitismo difere entre as famílias botânicas (Benzing 1990) e na Praia do Sul a maior parte das famílias apresenta espécies holoepífitas verdadeiras ou facultativas, com adaptações para o epifitismo durante todo o ciclo de vida. As hemiepífitas estão relacionadas, sobretudo, a Araceae, especialmente ao gênero Philodendron, cujas espécies se caracterizam por esse hábito (Benzing 1990).

Espécies são consideradas epífitas acidentais quando não apresentam adaptações estruturais e fisiológicas para viverem no dossel florestal, mas mesmo assim são encontradas sobre as árvores (Benzing 1990; Burns 2010). O epifitismo proporciona às espécies a oportunidade de se estabelecerem livres da competição com outras plantas do solo e expandirem a sua distribuição e nicho localmente (Guzmán-Marín \& Saldaña 2017). Anthurium intermedium e Neomarica candida são espécies essencialmente terrícolas, bastante abundantes na área de estudo e ocorreram como epífitas no tronco de árvores. Além da competição, a colonização dos forófitos pode estar relacionada à acidentes dispersivos associados a acumulação de matéria orgânica em decomposição nos troncos (Guzmán-Marín \& Saldaña 2017), o que permite que estas plantas cresçam e sobrevivam, apesar de não apresentarem especializações morfofisiológicas ao epifitismo (Zotz \& List 2003). Segundo Waechter (1998) alguns aspectos ambientais das matas de restinga, como clima úmido e solo bem drenado, favorecem que algumas espécies ocupem outros substratos disponíveis, como é o caso de plantas tipicamente terrícolas que colonizam acidentalmente troncos de árvores.

Os resultados para polinização e dispersão das espécies reforçam a importância das epífitas para manutenção dos recursos alimentares da fauna nos ecossistemas, já destacado por outros autores (Gentry \& Dodson 1987; Cruz-Angón \& Greenberg 2005; Dettke et al. 2008). A entomofilia possui grande destaque na comunidade epifítica da Mata Atlântica (Gentry \& Dodson 1987; Borgo \& Silva 2003; Cervi \& Borgo 2007; Hefler \& Faustione 2004; Oliveira et al. 2013) e ocorre principalmente em Orchidaceae, onde as 
especializações para atração de polinizadores são bem conhecidas (Gentry \& Dodson 1987). Em Araceae, o odor é um fator primordial para atração dos polinizadores, sobretudo abelhas e besouros (Mayo et al. 1997). Já a ornitofilia foi registrada apenas em bromélias, síndrome bastante comum na família e que ocorre especialmente através de beija-flores (Gentry \& Dodson 1987; Benzing 2000). Em relação à dispersão, o predomínio da zoocoria está principalmente relacionado à família Araceae. Muitos gêneros dessa família, como Anthurium e Philodendron, apresentam bagas que são dispersas principalmente por aves (ornitocoria) e mais raramente por mamíferos (mamaliocoria), morcegos ou primatas (Mayo et al. 1997). A zoocoria pode estar associada à ingestão dos diásporos pelos animais, comum nas famílias Araceae, Cactaceae e algumas Bromeliaceae, ou com a adesão dos diásporos à sua pele, típico de Piperaceae (Waechter 1992). Já a anemocoria, principal tipo de dispersão das epífitas segundo alguns estudos (Gentry \& Dodson 1987; Cervi \& Borgo 2007), pode ocorrer através de diásporos diminutos, encontrados principalmente em orquídeas e pteridófitas (Gentry \& Dodson 1987), ou por sementes maiores com apêndices plumosos, sobretudo em bromélias da subfamília Tillandsioideae (Waechter 1992), nos gêneros Tillandsia e Vriesea.

A Mata Atlântica é o quarto hotspot da biodiversidade mundial devido à elevada riqueza e excepcional taxa de endemismos (Myers et al. 2000; Mittermeier et al. 2004). Para epífitas vasculares, os valores são ainda mais elevados que para qualquer outro grupo biológico e as suas florestas abrigam 10\% das espécies conhecidas no planeta (Freitas et al. 2016). São registradas 2.256 espécies para a Mata Atlântica, com $78 \%$ delas endêmicas (Freitas et al. 2016). A alta riqueza e endemismos ocorrem devido a condições favoráveis de umidade atmosférica, temperatura e precipitação (Benzing 1990; Leitman et al. 2015; Menini Neto et al. 2016; Freitas et al. 2016), bem como a complexidade topográfica e heterogeneidade de habitats oferecida pelas florestas (Leitman et al. 2015; Freitas et al. 2016). A Mata Atlântica é o centro de dispersão e diversidade das famílias Araceae, Bromeliaceae, Polypodiaceae e Orchidaceae (Tryon 1986; Mayo et al. 1997; Siqueira-Filho \& Félix 2006; Martinelli et al. 2008), as mais ricas na flora epifítica, o que eleva o número de endemismos em todos os ecossistemas associados, inclusive restingas.
Nenhuma das epífitas da RBEPS encontra-se nas listas de espécies ameaçadas de extinção. No entanto, o estado de conservação é desconhecido para a maioria das espécies, já que não foram avaliadas em relação ao grau de ameaça (CNCFlora 2017). Epífitas são plantas sensíveis e com taxas de crescimento lento, representando um dos grupos mais vulneráveis nos ecossistemas e sendo indicadores adequados de mudanças na estrutura e no clima dos ambientes (Hietz 1998). Por esta razão, a comunidade epifítica pode ser empregada na avaliação do estado de conservação das florestas (Bonnet et al. 2009). A Praia do Sul possui uma restingas mais bem preservadas do estado do Rio de Janeiro (Rocha et al. 2007) e embora apresente vários pontos com vegetação secundária (Araujo \& Oliveira 1988), a sua mata abriga mais de $20 \%$ da riqueza total de epífitas das restingas do estado, 14,5\% das epífitas da Ilha Grande (213 espécies, Cruz \& Nunes-Freitas 2017) e é similar a registrada em outros estudos realizados em restingas (Waechter 1998; Foutoura et al. 2009; Becker et al. 2013; Graeff et al. 2015; Couto et al. 2017). A baixa riqueza de epífitas nas restingas do Rio de Janeiro está associada ao grande número de espécies terrestres de orquídeas e bromélias nesses ambientes, diferente do observado nas restingas do sul do país (Araujo 2000).

A conservação e a gestão adequada da biodiversidade exigem o conhecimento e a compreensão sobre ela. O presente trabalho é o primeiro a abranger a comunidade epifítica na área de estudo e os nossos resultados enfatizam a sua importância taxonômica e ecológica nesse ambiente. Destacamos a necessidade da realização de pesquisas populacionais e eco-fisiológicas, a fim de gerar informações sobre o estado de conservação das espécies visando garantir a proteção efetiva das epífitas e das restingas, um dos ecossistemas mais frágeis e ameaçados da Mata Atlântica.

\section{Agradecimentos}

À Coordenação de Aperfeiçoamento de Pessoal de Nível Superior (CAPES); à Universidade Federal Rural do Rio de Janeiro (UFFRJ); ao Programa de Pós-graduação em Ciências Ambientais e Florestais (PPGCAF); ao Programa de Pesquisas em Biodiversidade (PPBio); ao Instituto Estadual do Ambiente (INEA), a concessão da licença de coleta n ${ }^{\circ} 61 / 2015$; ao Parque Estadual da Ilha Grande (PEIG); à Reserva Biológica Estadual da Praia do Sul (RBEPS); ao Herbário RBR da UFRRJ; e a todas as pessoas que contribuíram de forma direta 
e indireta para o desenvolvimento da pesquisa, especialmente nas identificações taxonômicas e análise dos dados, ao Dr. Tiago de A. Amorim, à Dra. Aline dos Santos Dias, ao Dr. Marcus A. Nadruz Coelho e ao Dr. Felipe Fajardo V.A. Barberena.

\section{Referências}

Adhikari YP, Fischer HS \& Fischer A (2012) Host tree utilization by epiphytic orchids in different landuse intensities in Kathmandu valley, Nepal. Plant Ecology 213: 1393-1412.

Alho CJR, Schneider M \& Vasconcellos LA (2002) Degree of threat to the biological diversity in the Ilha Grande State Park (RJ) and guidelines for conservation. Brazilian Journal of Biology 62: 375-385.

Araujo DSD (2000) Análise florística e fitogeográfica das restingas do estado do Rio de Janeiro. Tese de Doutorado. Universidade Federal do Rio de Janeiro, Rio de Janeiro. 176p.

Araujo DSD \& Lacerda LD (1987) A Natureza das Restingas. Ciência Hoje 6: 42-48.

Araujo DSD \& Oliveira RR (1988) Reserva Biológica da Praia do Sul (Ilha Grande, estado do Rio de Janeiro): lista preliminar da Flora. Acta Botanica Brasilica 1: 83-94.

Assis AM, Thomaz LD \& Pereira OJ (2004) Florística de um trecho de restinga no município de Guarapari, Espírito Santo, Brasil. Acta Botanica Brasilica 18: 191-201.

Barthlott W, Schmit-Neuerburg V, Nieder J \& Engwald S (2001) Diversity and abundance of vascular epiphytes: a comparison of secondary vegetation and primary montane Rain Forest in the Venezuelan Andes. Plant Ecology 152: 145-156.

Bataghin FA, Barros F \& Pires JSR (2010) Distribuição da comunidade de epífitas vasculares em sítios sob diferentes graus de perturbação na Floresta Nacional de Ipanema, São Paulo, Brasil. Revista Brasileira de Botânica 33: 501-512.

Bataghin FA, Fiori A \& Toppa RA (2008) Efeito de borda sobre epífitos vasculares em floresta ombrófila mista, Rio Grande do Sul, Brasil. O Mundo da Saúde 32: 329-338.

Bataghin FA, Pires JSR, Barros F \& Muller A (2017) Epífitas vasculares da Estação Ecológica Barreiro Rico, Anhembi, SP, Brasil: diversidade, abundância e estratificação vertical. Hoehnea [online] 44: 172-183.

Becker DFP, Cunha S, Marchioretto MS \& Schmitt JL (2013) Riqueza, estrutura comunitária e distribuição vertical de epífitos vasculares do Parque Natural Municipal Tupancy, Arroio do Sal, RS, Brasil. Pesquisas, Botânica 64: 127-139.

Benavides AM, Duque MAJ, Duivenvoorder JF, Vasco GAJ \& Callajas R (2005) A first quantitative census of vascular epiphytes in rain forests of Colombian
Amazonia. Biodiversity and Conservation 14: 739-758.

Benavides AM, Wolf JHD \& Duivenvoorden JF (2006) Recovery and succession of epiphytes in upper Amazonian fallows. Journal of Tropical Ecology 22: 705-717.

Benzing DH (1990) Vascular epiphytes. Cambridge University Press, Cambridge. 354p.

Benzing DH (2000) Bromeliaceae - profile of an adaptative radiantion. Cambridge University Press, Cambridge. 690p.

BFG - The Brazil Flora Group (2018) Brazilian Flora 2020: innovation and collaboration to meet Target 1 of the Global Strategy for Plant Conservation (GSPC). Rodriguésia 69: 1513-1527.

Bianchi JS, Bento CM \& Kersten RA (2012) Epífitas vasculares de uma área de ecótono entre as Florestas Ombrófilas Densa e Mista, no Parque Estadual do Marumbi, PR. Estudos de Biologia - Ambiente e Diversidade 34: 37-44.

Blum CT, Roderjan CV \& Galvão F (2011) Composição florística e distribuição altitudinal de epífitas vasculares da Floresta Ombrófila Densa na Serra da Prata, Morretes, Paraná, Brasil. Biota Neotropica 11: 141-159.

Bonnet A, Lavoranti OJ \& Curcio GR (2009) Epífitos vasculares no Corredor de Biodiversidade Araucária, bacia do Rio Iguaçu, Paraná, Brasil. Cadernos de Biodiversidade 6: 49-70.

Breier TB (2005) O epifitismo vascular em florestas do sudeste do Brasil. Tese de Doutorado. Universidade Estadual de Campinas, São Paulo. 139p.

Burns KC (2010) How arboreal are epiphytes? A null model for Benzing's classifications. New Zealand Journal of Botany 48: 185-191.

Cervi AC \& Borgo M (2007) Epífitos vasculares no Parque Nacional do Iguaçu, Paraná, (Brasil): levantamento preliminar. Fontqueria 55: 415-422.

CNCFlora - Centro de Conservação da Flora (2017) Portal. Disponível em <http://cncflora.jbrj.gov.br/ portal/>. Acesso em 3 agosto 2017.

Coelho MAN, Waechter JL \& Mayo SJ (2009) Revisão taxonômica das espécies de Anthurium (Araceae) Seção Urospadix Subseção Flavescentiviridia. Rodriguesia 60: 799-864.

Coelho MAN, Soares ML, Calazans LSB, Gonçalves EG, Andrade IM, Pontes TA, Sakuragui CM, Temponi LG, Buturi C \& Mayo S (2015) Araceae. In: Lista de espécies da flora do Brasil. Jardim Botânico do Rio de Janeiro. Disponível em $<$ http://floradobrasil. jbrj.gov.br/jabot/floradobrasil/FB5015>. Acesso em 29 julho 2019.

Couto DR, Uribbe FP, Jacques SSA, Fracisco TM \& Lopes RC (2017) Vascular epiphytes in the Grumari restinga, RJ: floristic and similarities between restingas in Eastern Brazil. Rodriguésia 68: 337-346.

Cruz ACR \& Nunes-Freitas AF (2017) Diversidade e conservação das epífitas vasculares da Ilha Grande, 
RJ. Anais $6^{\circ}$ Simpósio de Gestão Ambiental e Biodiversidade. Disponível em < http://itr.ufrrj.br/ sigabi/6o-anais-simposio-de-gestao-ambiental-ebiodiversidade-issn-2525-4928/>. Acesso em 3 março 2018.

Cruz-Angón A \& Greenberg R (2005) Are epiphytes important for birds in coffee plantations? An experimental assessment. Journal of Applied Ecology 42: 150-159.

Cunha I (2005) Desenvolvimento sustentável na costa brasileira. Revista Galega de Economia 14: 1-14.

Dettke GA, Orfrini AC \& Milaneze-Gutierre MA (2008) Composição florística e distribuição de epífitas vasculares em um remanescente alterado de floresta estacional semidecidual no Paraná, Brasil. Rodriguésia 59: 859-872.

Dias ML, Prezoto F, Abreu PF \& Neto LM (2014) Bromélias e suas principais interações com a fauna. CES Revista, Juiz de Fora 28: 3-16.

Dislich R \& Mantovani W (1998) A flora de epífitas vasculares da reserva da Cidade Universitária “Armando de Salles Oliveira” (São Paulo, Brasil). Boletim de Botânica da Universidade de São Paulo 17: 1-83.

Flora do Brasil 2020 em construção. Intituto de Pesquisas Jardim Botânico do Rio de Janeiro. Disponível em $<$ http://floradobrasil.jbrj.gov.br/>. Acesso em 17 agosto 2017.

Fontoura T, Rocca MA, Schilling AC \& Reinert F (2009) Epífitas da floresta seca da Reserva Ecológica Estadual de Jacarepiá, Sudeste do Brasil: relações com a comunidade arbórea. Rodriguésia 60: 171-185.

Fontoura T, Sylvestre LS, Vaz AMSF \& Vieira CM (1997) Epífitas vasculares, hemiepífitas e hemiparasitas da Reserva Ecológica de Macaé de Cima. In: Lima HC \& Guedes-Bruni RR (eds.) Serra de Macaé de Cima: diversidade florística e conservação em Mata Atlântica. Instituto de Pesquisas Jardim Botânico do Rio de Janeiro, Rio de Janeiro. Pp. 89-101.

Freitas J \& Assis AM (2013) Estrutura do componente epífito vascular em trecho de Floresta Atlântica na região serrana do Espírito Santo. Revista Árvore [online] 37: 815-823.

Freitas L, Salino A, Menini Neto L, Almeida TE, Mortara SR, Stehmann JR, Amorim AM, Guimarães EF, Coelho MN, Zanin A \& Forzza RC (2016) A comprehensive checklist of vascular epiphytes of the Atlantic Forest reveals outstanding endemic rates. PhytoKeys 58: 65-79.

Gentry AH \& Dodson CH (1987) Diversity and biogeography of neotropical vascular epiphytes. Annals of the Missouri Botanical Garden 74: 205233.

Giongo C \& Waechter JL (2004) Composição florística e estrutura comunitária de epífitos vasculares em uma floresta de galeria na depressão central do Rio Grande do Sul. Revista Brasileira de Botânica 27: 563-572.
Gonçalves CN \& Waechter JL (2002) Epífitos vasculares sobre espécimes de Ficus organensis isoladas no norte da planície costeira do Rio Grande do Sul: padrões de abundância e distribuição. Acta Botanica Brasilica [online] 16: 429-441.

Graeff V, Parode MF, Paz ML, Silva VRSP, Marchioretto MS \& Schmitt JL (2015) Composição, estrutura comunitária, distribuição vertical e horizontal da sinúsia epifítica em fragmento de restinga, no Rio Grande do Sul, Brasil. Pesquisas, Botânica 68: 239-255.

Guedes D, Barbosa LM \& Martins SE (2006) Composição florística e estrutura fitossociológica de dois fragmentos de floresta de restinga no município de Bertioga, SP, Brasil. Acta Botanica Brasilica 20: 299-311.

Guzmán-Marín R \& Saldaña A (2017) Contribución del epifitismo accidental a la distribución de especies de plantas vasculares en un bosque templado lluvioso. Gayana. Botánica 74: 226-228.

Hefler SM \& Faustioni P (2004) Levantamento florístico de epífitos vasculares do Bosque São Cristóvão, Curitiba, Paraná, Brasil. Revista Estudos de Biologia 26: 11-19.

Hietz P (1998) Diversity and conservation of epiphytes in a changing environment. International Union of Pure and Applied Chemistry 70: 1-11.

INEA - Instituto Estadual do Ambiente (2011) Parque Estadual da Ilha Grande: plano de manejo (fase 2). Resumo executivo. Instituto Estadual do Ambiente, Rio de Janeiro. 98p.

INEA - Instituto Estadual do Ambiente (2017) Biodiversidade e áreas protegidas. Disponível em <http://www.inea.rj.gov.br/Portal/Agendas/ BIODIVERSIDADEEAREASPROTEGIDAS/ UnidadesdeConservacao/INEA_008602>. Acesso em 4 março 2017.

Irume MV, Morais MLCS, Zartman CE \& Amaral IL (2013) Floristic composition and community structure of epiphytic angiosperms in a terra firme forest in central Amazonia. Acta Botanica Brasilica 27: 278-393.

Kersten RA (2010) Epífitas vasculares - histórico, participação taxonômica e aspectos relevantes, com ênfase na Mata Atlântica. Hoehnea 37: 9-38.

Kersten RA, Borgo M \& Silva SM (2009) Diversity and distribution of vascular epiphytes in an insular Brazilian coastal forest. Revista de Biologia Tropical 57: 749-759.

Kersten RA \& Kuniyoshi YS (2009) Conservação das florestas na bacia do Alto Iguaçu, Paraná - avaliação da comunidade de epífitas vasculares em diferentes estágios serais. Revista Floresta 39: 51-66.

Kersten RA \& Silva SM (2001) Composição florística e estrutura do componente epifítico vascular em floresta da planície litorânea na Ilha do Mel, Paraná, Brasil. Revista Brasileira de Botânica 24: 213-226.

Kersten RA \& Silva SM (2002) Florística e estrutura do componente epifítico vascular em Floresta 
Ombrófila Mista Aluvial do Rio Barigüi, Paraná, Brasil. Revista Brasileira de Botânica 25: 259-267.

Kersten RA \& Silva SM (2006) The floristic compositions of vascular epiphytes of a seasonally inundated forest on the coastal plain of Ilha do Mel island, Brazil. Revista de Biología Tropical 54: 935-942.

Krömer T, Gradstein SR \& Acebey A (2007) Diversidad y ecología de epífitas vasculares en bosques montanos primarios y secundarios de Bolivia. Ecología en Bolivia 42: 23-33.

Leitman P, Amorim AM, Sansevero JBB \& Forzza RC (2015) Floristic patterns of epiphytes in the Brazilian Atlantic Forest, a biodiversity hotspot. Botanical Journal of the Linnean Society 179: 587-601.

Madison M (1977) Vascular epiphytes: their systematic occurrence and salient features. Selbyana 2: 1-13.

Magnusson WE, Lima AP, Luizão RC, Luizão F, Costa FRC, Castilho CV \& Kinupp VF (2005) RAPELD: a modification of the Gentry method for biodiversity surveys in long-term ecological research sites. Biota Neotropica 5: 1-6.

Mania LF \& Monteiro R (2010) Florística e ecologia de epífitas vasculares em um fragmento de floresta de restinga, Ubatuba, SP, Brasil. Rodriguésia 61: 705-713.

Martinelli G \& Moraes MA (2013) Livro vermelho da flora do Brasil. Andrea Jakobsson. Instituto de Pesquisas Jardim Botânico do Rio de Janeiro, Rio de Janeiro. 1100p.

Martinelli G, Vieira CM, Gonzalez M, Leitman P, Piratininga A, Costa AF \& Forzza R (2008) Bromeliaceae da Mata Atlântica brasileira: lista de espécies, distribuição e conservação. Rodriguésia 59: 209-258.

Martins SE, Rossi L, Sampaio PSP \& Magenta MAG (2008) Caracterização florística de comunidades vegetais de restinga em Bertioga, SP, Brasil. Acta Botanica Brasilica 22: 249-274.

Mayo SJ (1988) Aspectos da evolução e da geografia do gênero Philodendron Schott (Araceae). Acta Botanica Brasilica 1: 27-40.

Mayo SJ, Bogner J \& Boyce PC (1997) The genera of Araceae. Royal Botanical Garden, London. 370p.

Menini Neto L, Furtado SG, Zappi DC, Oliveira-Filho AT \& Forzza RC (2016) Biogeography of epiphytic Angiosperms in the Brazilian Atlantic Forest, a world biodiversity hotspot. Brazilian Journal of Botany 39: 261-173.

Mittermeier RA, Gil RP, Hoffmann M, Pilgrim J, Brooks T, Mittermeier CG, Lamoreux J \& Fonseca GAB (2004) Hotspots revisited: Earth's biologically richest and most endangered ecoregions. CEMEX, Mexico City. 392p.

MMA - Ministério do Meio Ambiente (2014) Portaria $\mathrm{n}^{\circ} 443$ de 17 de dezembro de 2014. Disponível em $<$ http://www.mma.gov.br/biodiversidade/especiesameacadas-de-extincao/atualizacao-das-listas-deespecies-ameacadas $>$. Acesso em 28 dezembro 2016.
MusskopfEL (2006) Composição florística e distribuição ecológica de epífitos vasculares no Parque Estadual de Itapuã, Viamão, Rio Grande do Sul. Dissertação de Mestrado. Universidade Federal do Rio Grande do Sul, Porto Alegre. 60p.

Myers N, Mittermeier RA, Mittermeier CG, Fonseca GAB \& Kent J (2000) Biodiversity hotspots for conservation priorities. Nature 403: 853-858.

Nadkarni NM \& Matelson TJ (1992) Biomass and nutrient dynamics of epiphytic litter-fall in a Neotropical montane forest, Costa Rica. Biotropica 24: 24-30.

Nieder J, Engwald S \& Barthlott W (1999) Patterns of neotropical epiphyte diversity. Selbyana 20: 66-75.

Nieder J, Engwald S, Klawu M \& Barthlott W (2000) Spatial distribution of vascular epiphytes (including hemiepiphytes) in a lowland Amazonian rain forest (Surumoni crane plot) of southern Venezuela. Biotropica 32: 385-396.

Nunes-Freitas AF, Rocha-Pessoa TC, CogliattiCarvalho L \& Rocha CFD (2006) Bromeliaceae da restinga da Reserva Biológica Estadual da Praia do Sul: composição, abundância e similaridade da comunidade. Acta Botanica Brasilica 20: 709-717.

Obermuller FA, Freitas L, Daly DC \& Marcos Silveira (2014) Patterns of diversity and gaps in vascular (hemi-)epiphyte flora of Southwestern Amazonia. Phytotaxa 166: 259-272.

Oliveira LC, Padilha PT, Dalmolim EB, Azeredo TEV \& Citadini-Zanette V (2013) Componente epifítico vascular de um fragmento florestal urbano, município de Criciúma, Santa Catarina, Brasil. Biotemas (UFSC) 26: 33-44.

Oliveira RR \& Coelho Netto AL (2001) Captura de nutrientes atmosféricos pela vegetação na Ilha Grande, RJ. Pesquisa Botânica 51: 31-49.

Perleberg TD, Garcia EN \& Pitrez SR (2013) Epífitos vasculares em área com floresta estacional semidecidual, Pelotas, Rio Grande do Sul, Brasil. Ciência e Natura, Santa Maria 35: 65-73.

Pos ET \& Sleegers ADM (2010) Vertical distribution and ecology of vascular epiphytes in a lowland tropical rain forest of Brazil. Boletim Museu Paranaense Emílio Goeldi. Ciências Naturais 5: 335-344.

Proctor M, Yeo P \& Lack A (1996) The natural history of pollination. Harper-Collins Publishers, London. $479 \mathrm{p}$.

Puente ME \& Bashan E (1994) The desert epiphyte Tillandsia recurvata harbours the nitrogen-fixing bacterium Pseudomonas stutzeri. Canadian Journal of Botany 72: 406-408.

Quaresma AC \& Jardim MAG (2014) Floristic composition and spatial distribution of vascular epiphytes in the restingas of Maracanã, Brazil. Acta Botanica Brasilica 28: 68-75.

Real L (1983) Pollination biology. Academic Press, Orlando. 338p. 
Ribeiro DCA (2009) Estrutura e composição de epífitas vasculares em duas formações vegetais na Ilha da Marambaia - Mangaratiba, RJ. Dissertação de Mestrado. Universidade Federal Rural do Rio de Janeiro, Seropédica. 129p.

Rocha CFD, Bergallo HG, Alves MAS \& Van Sluys M (2003) A biodiversidade nos grandes remanescentes florestais do estado do Rio de Janeiro e nas restingas da Mata Atlântica. Editora Rima, São Carlos. 160p.

Rocha CFD, Bergallo HG, Van Sluys M, Alves MA \& Jamel C (2007) The remnants of restinga habitats in the brazilian Atlantic Forest of Rio de Janeiro state, Brazil: habitat loss and risk of disappearance. Brazilian Journal of Biology 67: 263-273.

Rocha CFD, Nunes-Freitas AF, Rocha-Pessôa TC \& Cogliatti-Carvalho L (2004) Habitat disturbance in Brazilian Coastal sand dune vegetation and present richness and diversity of bromeliad species. Vidalia 2: 50-56.

Sabagh LT, Dias RJP, Branco CWC \& Rocha CFD (2011) News records of phoresy and hyperphoresy among treefrogs, ostracods, and ciliates in bromeliad of Atlantic forest. Biodiversity and Conservation 20: 1837-1841.

Sakuragui CM (2001) Biogeografia de Philodendron seção Calostigma (Schott) Pfeiffer (Araceae) no Brasil. Acta Scientiarum 23: 561-569.

Siqueira Filho JA \& Félix LP (2006) Bromélias e Orquídeas. In: Porto KC, Tabarelli M \& Almeida-Cortez JS (org.) Diversidade biológica e conservação da floresta Atlântica ao norte do
Rio São Francisco. Ministério do Meio Ambiente, Brasília. Pp. 219-226.

Staudt MG, Lippert APU, Cunha S, Becker DFP, Marchioretto MS \& Schmitt JL (2012) Composição florística de epífitos vasculares do Parque Natural Municipal Tupancy, Arroio do Sal, RS, Brasil. Pesquisas, Botânica 63: 177-188.

Thomazi RD, Rocha RT, Oliveira MV, Bruno AS \& Silva AG (2013) Um panorama da vegetação das restingas do Espírito Santo no contexto do litoral brasileiro. Natureza on line 11: 1-6.

Tryon R (1986) Biogeography of species, with special reference to ferns. Botanical Review 52: 117-156.

Veloso HP, Rangel Filho ALR \& Lima JCA (1991) Classificação da vegetação brasileira, adaptada a um sistema universal. IBGE-DERMA, Rio de Janeiro. $124 \mathrm{p}$.

Waechter JL (1986) Epífitos vasculares da mata paludosa do Faxinal, Torres, Rio Grande do Sul, Brasil. Iheringia, série Botânica 34: 39-49.

Waechter JL (1992) O epifitismo vascular na planície costeira do Rio Grande do Sul. Tese de Doutorado. Universidade Federal de São Carlos, São Carlos. $163 p$.

Waechter JL (1998) Epifitismo vascular em uma floresta de restinga do Brasil Subtropical. Revista Ciência e Natura 20: 43-66.

Zotz G (2013) The systematic distribution of vascular epiphytes - a critical update. Botanical Journal of the Linnean Society 171: 453-481.

Zotz G \& List C (2003) Zufallsepiphyten - Pflanzen auf dem Weg nach oben? Bauhinia 17: 25-37. 